\title{
DAISY STRUCTURE IN DESARGUESIAN PROJECTIVE PLANES
}

\section{GABRIELA ARAUJO PARDO}

(Received 5 June 2000; revised 13 November 2000)

\author{
Communicated by B. McKay
}

\begin{abstract}
We distribute the points and lines of $P G\left(2,2^{n+1}\right)$ according to a special structure that we call the daisy structure. This distribution is intimately related to a special block design which turns out to be isomorphic to $P G(n, 2)$.

We show a blocking set of $3 q$ points in $P G\left(2,2^{n+1}\right)$ that intersects each line in at least two points and we apply this to find a lower bound for the heterochromatic number of the projective plane.

2000 Mathematics subject classification: primary 51E15, 51E20, 51E21, $05 \mathrm{C} 15$.

Keywords and phrases: Desarguesian projective plane, oval, hyperoval, projective space, finite field, block design, blocking set, heterochromatic number.
\end{abstract}

\section{Introduction}

1.1. Basic objectives In this paper we distribute the points and lines of the Desarguesian projective plane of order $2^{n+1}$, denoted $P G\left(2,2^{n+1}\right)$, according to a special structure that we call the daisy structure. The daisy structure distributes the points in three sets, using a family of hyperovals, and allows us to distribute the lines on a dual structure whose combinatorial scheme is controled by a special block design which turns out to be isomorphic to the projective space of dimension $n$ over $\mathbb{Z}_{2}$, denoted $P G(n, 2)$.

We use the daisy structure to prove two applications, the first one related to the blocking sets in $P G(2, q)$ ([7]). In the second one, we see $P G(2, q)$ as a $(q+1)$ hypergraph and prove that the heterochromatic number of $P G(2, q)$ is greater than or equal to $q^{2}-2 q+5([3])$.

(C) 2003 Australian Mathematical Society 1446-7887/03 \$A2.00+0.00 
1.2. Basic notation and definitions Let $(P, \mathscr{L})$ be a projective plane of order $q$, with point set $\mathbf{P}$ and line set $\mathscr{L}$. It is well known that $(\mathbf{P}, \mathscr{L})$ has $q^{2}+q+1$ points and that $\mathscr{L}$ has $q^{2}+q+1$ lines with $q+1$ points on every line. An oval of $\mathbf{P}$ is a nonempty set $\mathscr{O}$ of points no three of which are collinear such that through any point of $\mathscr{O}$ there is precisely one tangent (a line that intersects $\mathscr{O}$ in only one point). A hyperoval of $\mathbf{P}$ is a nonempty set $\mathscr{H}$ of points such that any line intersects $\mathscr{H}$ in 0 or exactly 2 points. It is easy to see that any oval in $\mathbf{P}$ has exactly $q+1$ points and any hyperoval has exactly $q+2$ points [8].

It is well known that if $\mathscr{O}$ is any oval in a projective plane of order even all tangents (one for each point) pass through a common point, the nucleus of $\mathscr{O}$ [9]. In particular the theorem of Quist [12] states that any oval in a projective plane of even order can be extended to a hyperoval, if we take the oval plus its nucleus [8].

We work with the projective planes of order $q$ constructed over finite fields and denoted by $P G(2, q)$. Recall that given a vector space $V$ of dimension 3 over the finite field $G F(q)$ of order $q$, we can construct the projective plane $P G(2, q)$ whose points are the 1-dimensional subspaces of $V$ and whose lines are the 2-dimensional subspaces of $V$, moreover, it is well known that $P G(2, q)$ is Desarguesian [8]. Examples of ovals in Desarguesian planes are provided by nondegenerate quadrics (conics). The theorem of Segre [8] states that in projective planes of odd order there are no other ovals. The conclusion of Segre's theorem does not remain true if the plane has even order. In this paper we work with the Desarguesian projective planes of order $q$ for $q=2^{n+1}$ and $n \geq 0$.

Recall that the projective space of dimension $n$ over $\mathbb{Z}_{2}$, denoted $P G(n, 2)$, is constructed in a similar way, that is, if $V$ is the vector space over $\mathbb{Z}_{2}$ of dimension $n+1$, then $P G(n, 2)$, is the set of subspaces of $V$. Moreover, the hyperplanes of $P G(n, 2)$ are the subspaces of dimension $n-1$. It is well known, that $P G(n, 2)$ is a symmetric and cyclic $(q-1, q / 2-1, q / 4-1)$-block design (Singer [1938]), for $q=2^{n+1}$, where the elements of the design are the points of $P G(n, 2)$ and the blocks are the hyperplanes.

\section{The daisy structure in $P G(2, q)$}

2.1. The daisy structure of points Our first goal is to distribute the points in $P G(2, q)$ in a structure that resembles a daisy; we call it the daisy structure.

Since $G F^{*}(q)=G F(q)-\{0\}$ is a cyclic group [10] we can see $G F(q)$ like $\left\{0, \alpha^{0}, \ldots, \alpha^{q-2}\right\}$. It is easy to prove that there exist $(q-2) / 2$ different decompositions of the unit $1=\alpha^{a_{i}}+\alpha^{a_{i}+i}$, with $i \in\{1, \ldots,(q-2) / 2\}$ and $a_{i} \in\{1, \ldots, q-2\}$ ([2]).

Now, let us consider the following set of hyperovals in $P G(2, q)$

$$
\mathscr{H}_{i}=\left\{[x, y, z] \in \mathbf{P}: z^{2}=\alpha^{i} x y\right\} \cup\{[1,0,0]\} \text {, for each } i \in\{0, \ldots, q-2\} \text {. }
$$




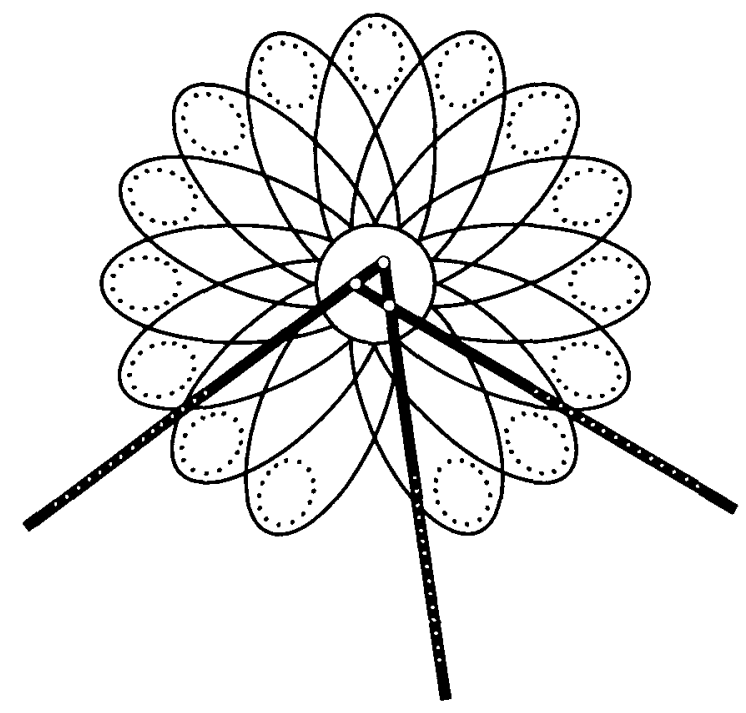

FIGURE 1. Daisy structure of points in $P G(2,16)$.

Equivalently, we can define $\mathscr{H}_{i}$ as

$$
\mathscr{H}_{i}=\{[1,0,0],[0,1,0],[0,0,1]\} \cup\left\{\left[1, a^{2} / \alpha^{i}, a\right] \backslash a \in G F^{*}(q)\right\} .
$$

The daisy's centre is the set $\mathscr{C}=\{[1,0,0],[0,1,0],[0,0,1]\}$, and we call it simply the centre.

It is easy to prove that two different hyperovals $\mathscr{H}_{i}$ and $\mathscr{H}_{j}$ for $i \neq j$ intersect exactly in the centre, it is $\mathscr{H}_{i} \cap \mathscr{H}_{j}=\mathscr{C}$.

The daisy's petals are the $q-1$ sets of points $\mathscr{P}_{i}=\mathscr{H}_{i}-\mathscr{C}$ for $i \in\{0, \ldots, q-2\}$, and we call them simply the petals. Observe that any petal has $q-1$ points and that the intersection of any two different petals is empty. The $q-1$ points of a petal $\mathscr{P}_{i}$ are $p_{i}^{j}=\left[1, \alpha^{2 j} / \alpha^{i}, \alpha^{j}\right]$ for $j \in\{0, \ldots, q-2\}$. Thus, we have $q^{2}-2 q+1$ points in the petals.

The daisy's stems are the points in the lines generated by $\mathscr{C}$ without the set $\mathscr{C}$. Thus, we have three stems with $q-1$ points in each one. Obviously two different stems have empty intersection, therefore we have $3(q-1)$ points of $P G(2, q)$ in the stems.

If we add the points in the centre, the petals and the stems we obtain all the points in $P G(2, q)$.

2.2. The daisy structure of lines In this section we distribute the lines of $P G(2, q)$ in a daisy structure of lines, dual to the daisy structure of points. We can observe that, by duality, an hyperoval (of lines) of $\mathscr{L}$ is a nonempty set $\mathscr{H}^{*}$ of lines such that any 
point in a line in $\mathscr{H}^{*}$ has exactly two lines in $\mathscr{H}^{*}$. In the following, we only use the words centre, petal and stem when we speak about these sets in the daisy structure of points and we shall be more specific otherwise.

We can observe that the daisy structure of points induce a natural partition of the lines in $P G(2, q)$ into three sets: the lines that meet the centre in two points, that meet the centre in one point and that do not meet the centre. In the following we construct with these sets the centre, the petals and the stems of the daisy structure of lines.

The daisy's centre of lines is the set of lines that meet two points in the centre. In other words it is $\mathscr{C}^{*}$.

The daisy's petals of lines are formed by the lines that do not meet the centre. Moreover, we have $q-1$ petals of lines $\mathscr{P}_{i}^{*}$ for $i \in\{0, \ldots, q-2\}$ and any $\mathscr{P}_{i}^{*}$ has $q-1$ lines $l_{i}^{j}$ such that $l_{i}^{j}=\left[1, \alpha^{2 j} / \alpha^{i}, \alpha^{j}\right]$. Thus, we have $q^{2}-2 q+1$ lines in the petals, it is easy to see that the lines of the petals of lines meet each line of $\mathscr{C}^{*}$ in one point and that this point is not part of the centre; therefore it is on the stem. Thus, we have that any line of the petals of lines has a point in each stem and the remaining $q-2$ points are in the petals. In this form any line of the petals of lines meets $(q-2) / 2$ petals in two points at each one.

The daisy's stems of lines are formed for the lines that meet the centre in only one point. Thus, by definition of hyperoval, any line in the stems of lines meets the $q-1$ petals in one point in each one, therefore the other point in these lines is in the stems.

We distribute these lines in three packets or stems of lines. Two lines are in the same stem if they meet the same point of the centre. Remember that in any point of the centre are $q+1$ lines, thus two of them are in $\mathscr{C}^{*}$ and the rest forms a stem of lines. It is easy to see that if $c \in \mathscr{C}$ and $T_{c}$ is the stem that does not meet $c$, then the $q-1$ lines in the stem of lines for $c$ meet each one of the points in $T_{c}$ and do not meet points in the others stems. Then we can identify the $3(q-1)$ lines in the stem of lines with the $3(q-1)$ points in the stems.

\section{The daisy block design}

We say that a line $l \in \mathscr{L}$ jumps $k$ in the petal $\mathscr{P}_{i}$ if it meet the points $p_{i}^{j}$ and $p_{i}^{j+k}$ for $j \in\{0, \ldots, q-2\}$, for a fixed $k \in\{1, \ldots,(q-2) / 2\}$. We denote by $S_{i}^{k}$ the set of these lines.

Let $\left\{a_{1}, \ldots, a_{(q-2) / 2}\right\}$ be the exponents of the $(q-2) / 2$ decompositions of unity $1=\alpha^{a_{k}}+\alpha^{a_{k}+k}$ introduced in the Section 2.1. We have the following.

Proposition 3.1. For a fixed $k \in\{1, \ldots,(q-2) / 2\}$, we have that $S_{i}^{k}=\mathscr{P}_{-i-2 a_{k}-k}^{*}$.

Proof. The line in $\mathscr{P}_{i}^{*}$ that meets the points $p_{i}^{j}$ and $p_{i}^{s}$ for $i, j, s \in\{0, \ldots, q-2\}$ 
and $j \neq s$ is $\left[\alpha^{j+s}, \alpha^{i}, \alpha^{j}+\alpha^{s}\right]$ and there is the cross product $p_{i}^{j} \times p_{i}^{s}$ with

$$
p_{i}^{j}=\left[1, \alpha^{2 j} / \alpha^{i}, \alpha^{j}\right] \text { and } p_{i}^{s}=\left[1, \alpha^{2 s} / \alpha^{i}, \alpha^{s}\right] .
$$

If we fix $k \in\{1, \ldots,(q-2) / 2\}$, the lines in $S_{i}^{k}$ meet the points $p_{i}^{s+a_{k}}$ and $p_{i}^{s+a_{k}+k}$ for $s \in\{1, \ldots, q-2\}$, therefore the lines in $S_{i}^{k}$ are $\left[\alpha^{2 s+2 a_{k}+k}, \alpha^{i}, \alpha^{s+a_{k}}+\alpha^{s+a_{k}+k}\right]$, for $s \in\{0, \ldots, q-2\}$. The decomposition of unity $1=\alpha^{a_{k}}+\alpha^{a_{k}+k}$ induce a decomposition of $\alpha^{s}$

$$
\alpha^{s}=\alpha^{s+a_{k}}+\alpha^{s+a_{k}+k}
$$

Therefore the lines in $S_{i}^{k}$ are

$$
\left[\alpha^{2 s+2 a_{k}+k}, \alpha^{i}, \alpha^{s}\right]=\left[1, \alpha^{i-2 s-2 a_{k}-k}, \alpha^{-s-2 a_{k}-k}\right]=\left[1, \frac{\alpha^{-2 s-4 a_{k}-2 k}}{\alpha^{-i-2 a_{k}-k}}, \alpha^{-s-2 a_{k}-k}\right] .
$$

Thus $S_{i}^{k}=\mathscr{P}_{-i-2 a_{k}-k}^{*}$.

In the next part we construct, via the daisy structure and Proposition 1, a symmetric and cyclic $(q-1, q / 2-1, q / 4-1)$-design that we call daisy block design and denote as $\mathscr{D} \mathscr{B} \mathscr{D}$. The elements of $\mathscr{D} \mathscr{B} \mathscr{D}$ are the petals of lines and its blocks are the petals.

The $\mathscr{D} \mathscr{B} \mathscr{D}$ is shown in the following table, whose $i j$-entry is the petal of lines that jumps $j$ in $\mathscr{P}_{i}$ :

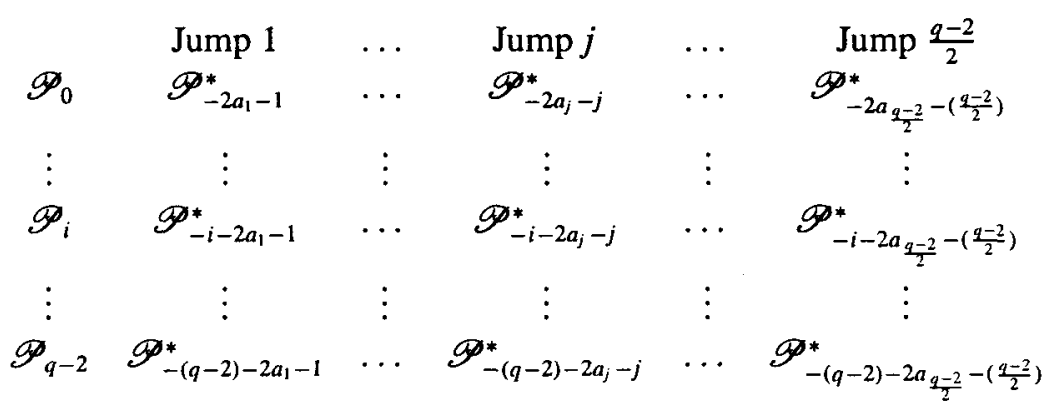

It is easy to prove, from many results about ovals [2], that each pair of petals of lines in $P G(2, q)$ meets together exactly $q / 4-1$ petals. Moreover, it is easy to see that this design is cyclic since its incidence matrix is circulant [11]. Observe that the $(n+1)$-row in the table is obtained subtracting one (in the subscript) from the $n$-row.

Now we have the following result.

THEOREM 3.2. There exists an isomorphism between the $\mathscr{D} \mathscr{B} \mathscr{D}$ constructed for $P G(2, q)$ and $P G(n, 2)$, where the points correspond to the petals of lines and the hyperplanes correspond to the petals. 
PROOF. Let $\mathscr{M}$ be the design isomorphic to $\mathscr{D} \mathscr{B} \mathscr{D}$ obtained by changing the signs of the subscript of the petals of lines in $\mathscr{D} \mathscr{B} \mathscr{D}$. $\mathscr{M}$ is, by construction, a symmetric block design with circulant incidence matrix.

Let $M$ be the design isomorphic to $\mathscr{M}$ whose elements are $\mathbb{Z}_{q}$ and the isomorphism $g: \mathscr{M} \rightarrow M$ is $g\left(\mathscr{P}_{i}^{*}\right)=i$ for all $i$. Obviously, $M$ is also isomorphic to $\mathscr{D} \mathscr{B} \mathscr{D}$ and it is a cyclic block design.

Now, let $\mathscr{N}$ be the cyclic block design isomorphic to $M$ whose elements are in $G F^{*}(q)$, and $\sigma: M \rightarrow \mathscr{N}$ is the isomorphism given by $\sigma(i)=\alpha^{i}$, where $\alpha$ is a primitive element in $G F^{*}(q)$ [10]. Again, by construction, $\mathscr{N}$ is isomorphic to $\mathscr{D} \mathscr{B} \mathscr{D}$. Recall that the first block in $\mathscr{D} \mathscr{B} \mathscr{D}$ has the petals of lines

$$
\left\{\mathscr{P}_{-2 a_{j}-j}^{*} \mid j \in\{1, \ldots,(q-2) / 2\}\right\} \text {. }
$$

Therefore the first block in $M$ is

$$
\left\{2 a_{j}+j \mid j \in\{1, \ldots,(q-2) / 2\}\right\} .
$$

Thus, the first block in $\mathscr{N}$ is

$$
\mathbf{B}=\left\{\alpha^{2 a_{j}+j} \mid j \in\{1, \ldots,(q-2) / 2\}\right\}
$$

when $\alpha$ is a primitive element of $G F^{*}(q)$.

Now, we will prove that $\mathbf{B} \cup\{0\}$ is a subgroup of $G F(q)$. Note that $\alpha^{a_{i}}\left(1+\alpha^{i}\right)=1$ and then $\alpha^{2 a_{i}+i}=\alpha^{i} / 1+\alpha^{2 i}$. Also,

$$
\frac{\alpha^{i}}{1+\alpha^{2 i}}=\frac{\alpha^{-i}}{\alpha^{-2 i}+1}
$$

Thus, we have proved that

$$
\mathbf{B}=\left\{\frac{\alpha^{i}}{1+\alpha^{2 i}} \mid i \in\{1, \ldots,(q-2) / 2\}\right\} .
$$

Recall that, if $i \in\{1, \ldots, q-1\}$, there always exists an $s \in\{1, \ldots, q-1\}$ such that $\alpha^{i}+\alpha^{s}=1$. Therefore, $\alpha^{i} /\left(1+\alpha^{2 i}\right)=\alpha^{-s}\left(1+\alpha^{-s}\right)$, because

$$
\frac{\alpha^{i}}{1+\alpha^{2 i}}=\frac{\alpha^{s}+1}{1+\alpha^{2 s}+1}=\frac{\left(1+\alpha^{-s}\right) / \alpha^{-s}}{1 / \alpha^{-2 s}}=\alpha^{-s}\left(1+\alpha^{-s}\right)
$$

Let $\alpha^{i} /\left(1+\dot{\alpha}^{2 i}\right)$ and $\alpha^{j} /\left(1+\alpha^{2 j}\right)$ be in $\mathbf{B}$, then there exist $s$ and $k \in\{1, \ldots, q-1\}$ such that:

If we add two elements of $B$ we have that

$$
\frac{\alpha^{i}}{1+\alpha^{2 i}}+\frac{\alpha^{j}}{1+\alpha^{2 j}}=\alpha^{-s}+\alpha^{-k}\left(1+\alpha^{-s}+\alpha^{-k}\right)
$$


Moreover, we know that $\alpha^{-s}+\alpha^{-k}=\alpha^{p}$ for some $p \in\{1, \ldots, q-1\}$, and that there exists $r \in\{1, \ldots, q-1\}$ such that $\alpha^{p}+\alpha^{r}=1$, therefore

$$
\alpha^{-s}+\alpha^{-k}\left(1+\alpha^{-s}+\alpha^{-k}\right)=\alpha^{p}\left(1+\alpha^{p}\right)=\frac{\alpha^{r}}{1+\alpha^{2 r}} .
$$

Thus, $\mathbf{B} \cup\{0\}$ is a subgroup of order $q / 2$ in $G F(q)$.

Singer's Theorem asserts that $P G(n, 2)$ is a cyclic $(q-1, q / 2-1, q / 4-1)$-design. Using the idea of the proof in the Singer's Theorem:

Let $f: P G(n, 2) \rightarrow G F^{*}(q)$ be such that $f\left[a_{n}, a_{n-1}, \ldots, a_{0}\right]=\sum a_{i} \alpha^{i}$ for $a_{i} \in \mathbb{Z}_{2}$ and $\alpha$ a primitive element of $G F^{*}(q)$. Obviously, $f$ is one to one and sends the hyperplanes to subsets in $G F^{*}(q)$, in which the exponents of $\alpha$ form a difference set [1].

Let $P G(n, 2) \cup\{0\}$ be $P G(n, 2) \cup\left\{\left[a_{n}, a_{n-1}, \ldots, a_{0}\right] \mid a_{i}=0 \forall i\right\}$. If we define $f(0)=0$; then $f$ sends $P G(n, 2) \cup\{0\}$ into $G F(q)$.

We know that $P G(n, 2) \cup\{0\}$ is the vector space of dimension $n+1$ over $\mathbb{Z}_{2}$ and $f$ sends the subspaces of dimension $q / 2$ to subgroups of $G F(q)$ of order $q / 2$.

Now, if $H$ is any hyperplane then $H \cup\{0\}$ is a subspace of dimension $q / 2$. Thus, for any $H$ hyperplane of $P G(n, 2)$, the image under $f$ of $H \cup\{0\}$ are the subgroups of $G F(q)$ of order $q / 2$. Then, $\mathbf{B}$ is the image of some hyperplane. Moreover, since $\mathscr{N}$ is the block design generated by $\mathbf{B}$, it is isomorphic to $P G(n, 2)$.

\section{Two applications of the daisy structure}

In this section we apply the results of the daisy structure and the daisy block design discussed in the previous sections. First to blocking sets of projective planes and then we use this application to find a lower bound for the heterochromatic number, introduced in [3], of the projective planes.

Remember that if $(\mathbf{P}, \mathscr{L})$ is a projective plane, a blocking set of $\mathbf{P}$ is a set $B$ of points such that any line contains a point of $B$ and a point outside $B$. Moreover, we know that if $B$ is a blocking set in a projective plane of order $q$, then

$$
\left.q+\sqrt{q}+1 \leq|B| \leq q^{2}-\sqrt{q} \quad \text { (Bruen }[7,6]\right) .
$$

Using the isomorphism between the daisy block design and $P G(n, 2)$ given in the last section we have the following theorem.

THEOREM 4.1. A Desarguesian projective plane of order $q=2^{n+1}$, for $n>1$, has a blocking set of $3 q$ points that intersects each line in at least two points.

PROOF. Let $\mathscr{D} \mathscr{B} \mathscr{D}^{*}$ be the cyclic and symmetric $(q-1, q / 2-1, q / 4-1)$-design dual to $\mathscr{D} \mathscr{B} \mathscr{D}$ whose elements are the petals of points and the blocks are the petals of 
lines such that an element $\mathscr{P}_{i}$ is in a block $\mathscr{P}_{j}^{*}$ if $\mathscr{P}_{j}^{*}=S_{i}^{k}$, for $k \in\{1, \ldots,(q-2) / 2\}$. It is easy to prove that $\mathscr{D} \mathscr{B} \mathscr{D}^{*}$ is isomorphic to $\mathscr{D} \mathscr{B} \mathscr{D}([1,2])$, therefore it is also isomorphic to $P G(n, 2)$ (Theorem 3.2). Let $f$ be the isomorphism between $\mathscr{D} \mathscr{B} \mathscr{D}^{*}$ and $P G(n, 2)$ and let $\mathscr{P}_{1}, \mathscr{P}_{2}$ and $\mathscr{P}_{3}$ be three petals of points whose images under $f$ are three collinear points in $P G(n, 2)$. Since three collinear points in a projective space are together in exactly $q / 4-1$ hyperplanes [1], we claim that $\mathscr{P}_{1}, \mathscr{P}_{2}$ and $\mathscr{P}_{3}$ are together in exactly $q / 4-1$ blocks of $\mathscr{D} \mathscr{B} \mathscr{D}^{*}$.

Moreover, we know that any pair of elements in $\mathscr{D} \mathscr{B} \mathscr{D}^{*}$ are together in exactly $q / 4-1$ blocks and that any element in $\mathscr{D} \mathscr{B} \mathscr{D}^{*}$ is exactly in $q / 2-1$ blocks then, it is easy to see that there exist $q / 4-1$ blocks with $\mathscr{P}_{1}, \mathscr{P}_{2}$ and $\mathscr{P}_{3}$ and that any other block in $\mathscr{D} \mathscr{B} \mathscr{D}^{*}$ is one and only one of the three petals of points $\mathscr{P}_{1}, \mathscr{P}_{2}$ and $\mathscr{P}_{3}$.

Now, since the petals of lines are the blocks in $\mathscr{D} \mathscr{B} \mathscr{D}^{*}$, it is easy to see that any line in the petals of lines (Section 2.2) meets some petal of points $\mathscr{P}_{i}$, for $i \in\{1,2,3\}$.

Consider the set of points $\mathscr{A}=\mathscr{H}_{1} \cup \mathscr{P}_{2} \cup \mathscr{P}_{3}$ in $P G(2, q)$, and recall that $\mathscr{H}_{1}=\mathscr{P}_{1} \cup \mathscr{C}$ (Section 2). We claim that $\mathscr{A}$ is a blocking set that satisfies the hypothesis of the theorem. Now, we will prove that any line in $P G(2, q)$ has at least two points in $\mathscr{A}$.

Let $l \in \mathscr{L}$. If $l$ is in the centre of lines (Section 2.2) it has two points in $\mathscr{H}_{1}$. If $l$ is a line of the petals of lines we proved that it has two points in at least one of three sets $\mathscr{P}_{1}, \mathscr{P}_{2}$ or $\mathscr{P}_{3}$ (observe that it is possible that $l$ has two points in each one of this sets). Now, if $l$ is a line of the stems of lines (Section 2.2) it has two points in $\mathscr{H}_{1}$, one point in $\mathscr{P}_{2}$ and one point in $\mathscr{P}_{3}$.

It is clear, (by the definition of $\mathscr{A}$ ) that all lines in $\mathscr{L}$ have more than one point outside of $\mathscr{A}$.

Finally, if we add the points in $\mathscr{H}_{1}, \mathscr{P}_{2}$ and $\mathscr{P}_{3}$, we find that $|\mathscr{A}|=3 q$.

Now, we will apply our results to Graph Theory; we see $P G(2, q)$ as a $(q+1)$ hypergraph, $H=(V, E)$, where $V=\mathbf{P}$ and $E=\mathscr{L}$ and prove that the heterochromatic number, introduced in [3], is greater than or equal to $q^{2}-2 q+5$.

Recall that, if $H=(V ; E)$ is a hypergraph, by a $t$-coloring of $H$ we mean a surjective mapping from the vertex set $V$ onto a $t$-element set. A $t$-coloring $f$ of $H$ separates the edge $\alpha \in E$ if the images by $f$ of the vertices in $\alpha$ are all different. We call $f$ heterochromatic if $f$ separates some edge of $H$. The heterochromatic number of $H$, denoted $h_{c}(H)$, is the maximum $t$ for which there exists a $(t-1)$-coloring that is not heterochromatic.

Then we prove the following theorem.

THEOREM 4.2. For $q=2^{n+1}$ and $n \geq 1$ we have that $h_{c}(P G(2, q)) \geq q^{2}-2 q+5$.

Proof. We will construct, using Theorem 4.1, a $q^{2}-2 q+4$-coloring that is not heterochromatic. If we have the sets of points $\mathscr{H}_{1}, \mathscr{P}_{2}$ and $\mathscr{P}_{3}$ as in Theorem 4.1 
and we let $f: \mathbf{P} \rightarrow\{1, \ldots, t\}$ be a $t$-coloring of $P G(2, q)$ such that $f\left(\mathscr{H}_{1}\right)=1$, $f\left(\mathscr{P}_{2}\right)=2$ and $f\left(\mathscr{P}_{3}\right)=3$ and the rest of the points in $\mathbf{P}$ has each one a different color and also different to 1,2 and 3 we have a $t$-coloring that is not heterochromatic since any line of $P G(2, q)$ has at least two points in any of these three sets. Moreover, we have that $t=3+\left(q^{2}+q+1-3 q\right)=q^{2}-2 q+4$.

It is important to point out that we can prove that this bound is exact for $P G(2,4)$.

We also proved that the heterochromatic number for any projective plane or order $q$ is greater than or equal to $q^{2}-2 q+4$ and it has as upper bound the number $q^{2}-q+1$. These results are obtained using the concept of trace in a hypergraph [4] and blocking sets. Details will appear elsewhere.

\section{References}

[1] I. Anderson, Combinatorial designs. Construction methods (Ellis Horwood, Chinchester, 1990).

[2] G. Araujo, La Margarita. Un estudio acerca de la estructura y la distribución de las rectas de los Planos Proyectivos Algebraicos $P G\left(2,2^{n}\right)$ (Ph.D. Thesis, Facultad de Ciencias, UNAM, 2000).

[3] J. L. Arocha, J. Bracho and V. Neumann-Lara, 'On the minimum size of tight hypergraphs', J. Graph Theory 16 (1992), 319-326.

[4] —_, 'Tight and untight triangulated surfaces', J. Combin. Theory Ser. B 63 (1995), 319-326.

[5] L. M. Batten, Combinatorics of finite geometries (Cambridge University Press, Cambridge, 1986).

[6] A. A. Bruen, 'Blocking sets in finite projective planes', SIAM J. Appl. Math 21 (1971), 380-392.

[7] A. A. Bruen and J. A. Thas, 'Blocking sets', in: Geometriae dedicata, 6 (Reidel, Dordrecht, 1977) pp. 193-203.

[8] F. Buekenhout, Handbook of incidence geometry (Université Libre de Bruxelles, Belgium, 1995).

[9] J. W. P. Hirschfeld, Projective geometries over finite fields (Clarendon Press, Oxford, 1979).

[10] R. Lidl and H. Niederreiter, Finite fields, Encyclopedia Math. Appl. 20 (Cambridge University Press, Cambridge, 1977).

[11] H. J. Ryser, Combinatorial mathematics, volume 14 (Quinn \& Boden Company, New Jersey, 1963).

[12] W. D. Wallis, Combinatorial designs (Dekker, 1988).

Instituto de Matemáticas, UNAM

Circuito Exterior

Ciudad Universitaria

México 04510 D.F.

México

e-mail: garaujo@math.unam.mx 
